\title{
Contralateral Breast Carcinoma
}

National Cancer Institute

\section{Source}

National Cancer Institute. Contralateral Breast Carcinoma. NCI Thesaurus. Code C99390.

Breast carcinoma that develops in the opposite breast of a patient with an already diagnosed primary breast carcinoma. 